\title{
Tibial Alignment in Total Knee Replacement Surgery, Intramedullary Alignment Versus Extramedullary Alignment a Blinded Single Surgeon Study
}

\author{
Mr Walaa El Nahas", Mr Iheanyi Nwachuku, Mr Khalid Khan, Ayman Gabr and Mr John \\ Hodgkinson
}

\author{
Wrightington Hospital, Julia Berry, MCh Course Administrator, Hall Lane, Appley Bridge, Wigan, Lancashire WN6 9EP, UK
}

\begin{abstract}
Background: The clinical success of total knee arthroplasty is positively correlated with good component orientation. Controversy remains as to what is the best tibial alignment technique, this study compares the intramedullary versus the extramedullary surgical alignment guidance methods.

Material and Methods: A retrospective study was done on 100 patients during the time period 2007 to 2010 . The knee replacements were performed by the same surgeon, 49 procedures were performed via the intramedullary technique and 51 procedures were via the extramedullary technique. The tibiofemoral angle was measured pre-operatively as well as post operatively, the tibial alignment angle was measured post operatively also, the results were then statistically analysed.

Results and Conclusion: There was no significant difference between both groups regarding the tibial alignment angles. Both techniques proved accurate in producing an acceptable post operative tibial component alignment angle. We advocate the surgeon may use his/her discretion when deciding upon the method of tibial alignment.
\end{abstract}

Keywords: Extramedullary, intramedullary, Tibial alignment.

\section{INTRODUCTION}

The world of total joint replacement arthroplasty strives to continue to improve in efficacy, efficiency, longevity, function and to improve the re-operation rates. These goals are no different when considering total knee replacement surgery. Total knee replacement (TKR) surgery is a well recognised surgical treatment option for the advanced stages in the process of rheumatoid or osteoarthritis. At present orthopaedics surgeons are treating a much younger population in need of a total knee replacement, it is not uncommon to see patients in their mid- 30's are requiring total knee arthroplasty. This younger population has prompted the authors to propose a study to determine the best technique for alignment of the tibial prosthesis. It is known that the femoral prosthetic alignment has been proven to be superior via the intramedullary method [1] however this is not the case for the tibia. It is also known that the overall alignment of the complete prosthesis governs the longevity of the implants and also governs the function a patient will experience. Accurate implant alignment is crucial particularly in younger more active patients [2].

Important angles of alignment for total knee replacement described in the literature [3] are:

1. Tibial femoral angle, an angle formed from the angle subtended between the femoral and tibial anatomic axis. Normal values 5-10 degrees.

*Address correspondence to this author at the Wrightington Hospital, Julia Berry, MCh Course Administrator, Hall Lane, Appley Bridge, Wigan, Lancashire WN6 9EP, UK; Tel: 00447533737693 ;

E-mail:walaa_nahas@yahoo.com
2. The tibial component or the tibial tangent angle, the angle formed between a tangent to the tibial component and the tibial axis. Normal 90 degrees.

3. The femoral component angle, the angle formed between a tangent to the end of the femoral component and a line perpendicular to the femoral axis. Normal 5 degrees.

The senior author $(\mathrm{JH})$ has a long standing practice of over twenty years at consultant level offering lower limb arthroplasty. All the early total knee replacements completed by $\mathrm{JH}$ initially were performed using the extramedullary method up until May 2009. Outside influences and peers in the field of lower limb arthroplasty encouraged JH to change technique. Thus $\mathrm{JH}$ reverted to the intramedullary method for the tibial alignment. After the generation of a significant number of participants, a retrospective study was performed in our centre to determine which method was superior in terms of alignment. This is a valuable study as we have controlled the differences in operating surgeon, operating centre and experience (which is seen in previous studies) by using a single surgeon and a single centre for all cases.

\section{MATERIALS AND METHODS}

For this retrospective data collecting study we declare all patients details are anonymous, the study is authorized by the local ethical committee and was performed in accordance with the ethical standards of the 1964 declaration of Helsinki as revised in 2000 .

This is a single centre retrospective study; the senior author $(\mathrm{JH})$ undertook a total of 100 total knee replacements 
Table 1. Descriptive Statistics

\begin{tabular}{|c|c|c|c|c|c|c|c|c|}
\hline Variable & Group & $\mathbf{N}$ & Mean & Median & SD & Min & Max & P Value \\
\hline \multirow{2}{*}{ Preoperative Tibio-Femoral Angle } & EM & 51 & 7.64 & 7.00 & 3.50 & 3.00 & 17.00 & \multirow{2}{*}{0.781} \\
\hline & IM & 39 & 7.17 & 6.00 & 3.72 & 1.00 & 15.00 & \\
\hline \multirow{2}{*}{ Post Operative Tibio-Femoral Angle } & EM & 51 & 2.90 & 3.00 & 1.55 & 1.00 & 6.00 & \multirow{2}{*}{0.829} \\
\hline & $\mathrm{IM}$ & 49 & 2.77 & 2.00 & 1.31 & 1.00 & 6.00 & \\
\hline \multirow{2}{*}{ Post Operative Tibial Tangent Angle } & EM & 51 & 87.45 & 88.00 & 1.70 & 84.00 & 90.00 & \multirow{2}{*}{0.143} \\
\hline & $\mathrm{IM}$ & 49 & 87.98 & 88.00 & 1.66 & 85.00 & 91.00 & \\
\hline
\end{tabular}

Descriptive Statistics. $\quad \mathrm{N}=$ Number participants $\mathrm{SD}=$ Standard Deviation

during the course of May 2007 to May 2010. 51 total Knee replacements were performed using an extramedullary technique for the tibial alignment occurring between May 2007 to August 2009. 49 total knee replacements were performed via an intramedullary technique, occurring over 9 months, between September 2009 to May 2010. The centre and the operating surgeon for both techniques have remained constant. The implant used was the same throughout all patients of both categories. The implant was the well established cemented PFC cruciate retaining arthroplasty system. The surgery was undertaken using the standard techniques as described by the manufacturers in the operative technique manuals via a median para patellar approach. A tourniquet was used for both methods of total knee replacement and the pre surgical set up was kept constant as was the surgical approach. The patients had the same rehabilitation of next day full weight bearing mobilisation and standard AP and Lateral new radiographs were taken at the first clinic appointment at 6 weeks.

The 6 week post operative films were retrospectively measured via the picture and archiving systems (PACS) digital radiographic software for the tibial femoral angles and the tibial tangent angles, (the Femoral component angles were not measured). The researcher measuring these angles had not been involved in the surgery and did not know which group the patients belonged to (therefore was blinded). The pre-operative standard length knee films were then retrospectively measured for the tibial femoral angles, thus allowing a pre-op and post operative comparison, a range of a 5-10 degree angle was taken as being normal. Therefore less than 5 degrees was considered a varus knee and greater than 10 degrees considered a valgus knee. A further comparison was made by also measuring a second angle of alignment, the tibial tangent angle as described above. The different groups were then statistically analysed using SPSS 16.0 for windows 2007 for any significant differences in the pre-operative tibial femoral angles and the post operative angles. A statistical analysis using the Mann Whitney $U$ test was also performed to compare the statistical differences in means between the post operative tibial tangent angles. Unfortunately ten pre op angles were missing from the intramedullary (IM) group as the data had not been collected well and was non retrievable at the time of writing the study. This has left the intramedullary group with only 39 measurements as seen in Table $\mathbf{1}$.

\section{RESULTS}

The radiographs of 100 included patients were evaluated in this study. The extramedullary group consisted of 51 patients over the period of nine months (from September 09 to May 2010). The average age was 71.9; there were 25 males and 26 females. The intramedullary group consisted of 49 patients over a period of 28 months (from May 2007 to August 2009). The average age was 69.5 years with 25 females and 24 males. For the extramedullary group there were 13 valgus knees 11 varus knees and 27 knees in neutral alignment. For the Intramedullary group there were 8 valgus 5 varus and 36 knees in neutral alignment pre-operatively. Therefore no sub group comparisons were performed as the subgroups were too small to take into separate consideration, also this shows that the preoperative measurements between the groups were of similar comparison.

The two groups were tested for homogeneity and were found to be non homogenous thus a Mann Whitney $U$ test was performed on all group comparisons. Initially the groups were tested for their individual pre-operative tibial femoral angles, no significant difference was found $(\mathrm{P}=0.781)$ between the groups pre-operatively, a $\mathrm{P}$ value of below 0.05 was taken to be significant. The comparisons between the measured post operative angles were also tested for statistical significance Via the Mann Whitney U tests and no significant differences were found here either. For the Tibial Femoral angles $\mathrm{P}=0.829$ and comparing the Tibial tangent angles $\mathrm{P}=0.143$.

\section{DISCUSSION}

In summary this study offers a unique comparison against two well used methods of tibial alignment when performing a total knee replacement. The direct comparison of the two methods was made whilst keeping the majority of other factors constant. The surgeon, the centre, implant materials, the approach and the post operative rehabilitation of the patients were all kept constant. The results obtained at our centre have directly compared the 2 differing methods and found very similar results regarding the two methods for TKR, whereby $13.7 \%$ (7/51) of the extramedullary TKR's achieved a perfect 90 degrees tibial tangent angle and $22.4 \%$ $(11 / 49)$ of the intramedullary achieved the perfect 90 degrees.

The limitations of the study include a small number sample size, use of the less accurate 'standard length' radiograph for review on PACS instead of the 'long leg' alignment view, this was done due to a study by Ishii et al [4] stated short radiographs are be accurate for such measurements. The final clear limitation was having incomplete data, the missing data for 10 sets of patients preoperative angles were in the extramedullary group this 
could have had an influence on the before surgery group comparisons.

The theoretical advantages and disadvantages of both intramedullary and extramedullary methods are well documented in the literature [5]. The intramedullary method cannot be accurately used in patients who have a history of skeletal trauma and subsequent deformity, neither can the intramedullary method be reliably used in those with retained metal work or used on those with significant natural bowing of the tibia. The intramedullary method also can compromise the effectiveness of the press-fit cementing technique, this is due to the relatively large space that is made on the tibial surface connecting with the medullary canal. Some surgeons opt to plug the space in order to strengthen the tibial platform. The extramedullary technique has also been criticised because of the inaccuracies with the surgeon estimating or 'eyeballing' where the centre of the tibial axis should be. It is also highly unreliable in obese patients where the centre of the talus is difficult to locate. Of note Lorenzo et al. [6] did not find obesity an issue and instead reported a reduced surgical time with intramedullary techniques as the tibial centre is found more readily.

The Debate between intramedullary and extramedullary tibial cutting Jigs/guides/ devices continues and most orthopaedic surgeons will use their own preferred technique and will continue to achieve good post operative results as we have found in our centre. Our study is rare due to the fact we have a single surgeon performing both techniques therefore controlling for any surgical experience or operating technique differences. The post operative radiographs were also reviewed at the $1^{\text {st }}$ outpatient clinic appointment, thus patient demographics are also controlled. This method ensures any differences in patient weight, rehabilitation or bone quality should not have any influence on the $1^{\text {st }}$ post op film, thus focussing on the operative technique only. The study has also blinded the $1^{\text {st }}$ researcher improving the validity and reducing any possible observer bias. We have found no significant difference between the intramedullary method for TKR tibial alignment and the extramedullary method, and would advocate Orthopaedic surgeons should remain at liberty to utilise their most familiar tibial alignment cutting technique, either an intra or extramedullary method, this is in keeping with similar studies in the literature $[7,8]$.

\section{CONFLICT OF INTEREST}

The authors confirm that this article content has no conflicts of interest.

\section{ACKNOWLEDGEMENT}

Declared none.

\section{REFERENCES}

[1] Cates HE, Ritter MA, Keating EM, Faris PM. Intramedullary versus extramedullary femoral alignment systems in total knee replacement. Clin Orthop Relat Res 1993; 286: 32-9.

[2] Bargren JH, Blaha JD, Freeman MA. Alignment in total knee arthroplasty. Correlated Biomechanical and clinical observations. Clin Orthop Relat Res 1983; 173: 178-83.

[3] Moreland JR, Bassett LW, Hanker, GJ. Radiographic Analysis of the axial alignment of the lower extremity. J Bone Joint Surg 1987; 69: 745-9.

[4] Ishii Y, Ohmori G, Bechtold JE, Sherman RE, Gustilo RB. Accuracy of the short radiograph in the measurement of the tibiofemoral angle. Knee 1995; 2(2): 81-4.

[5] Reed MR, Bliss W, Sher JL, Emmerson KP, Jones SMG, Partington PF. Extramedullary or intramedullary tibial alignment guides: a randomised, prospective trial of radiological alignment. J Bone Joint Surg Br 2002; 84-B: 858-60.

[6] Lorenzo LM, Segur JM, Macule F, et al. Intramedullary versus extramedullary tibial cutting guide in severely obese patients undergoing total knee replacement: A randomized study of 70 patients with body mass index $>35 \mathrm{~kg} / \mathrm{m}^{2}$. Obes Surg 2008; 1(8):1599-604.

[7] Rottman SJ, Dvorkin M, Gold D. Extra medullary versus intramedullary tibial alignment guides for total knee arthroplasty. Orthopaedics 2005; 28(12): 1445-8.

[8] Dennis DA, Channer M, Susman MH, Stranger EA. Intermedullary versus extra medullary tibial alignment systems in total knee arthroplasty. J Arthroplasty 1993; 8(1): 43-7. 